\title{
Relación imagenológica de la infección por COVID-19 con hipertensión arterial pulmonar en TC
}

\section{Imaging relationship between COVID-19 infection with pulmonary}

\section{arterial hypertension in CT}

Mario A. Cuevas-Bailón*, Edurne G. Cantero-Pérez y Johana P. Sánchez-Orjuela

Departamento de Imagenología Diagnóstica y Terapéutica del Hospital Regional General Ignacio Zaragoza, Instituto de Seguridad y Servicios Sociales de los

Trabajadores del Estado (ISSSTE), Ciudad de México, México

\section{RESUMEN}

Objetivo: Identificar en una muestra de pacientes con diagnóstico de infección por SARS-Cov-2 la relación del COVID-19 con los datos tomográficos de hipertensión arterial pulmonar (HAP), al mostrar un calibre del tronco de la arteria pulmonar (TAP) > $30 \mathrm{~mm}$ como manifestación indirecta de la HAP; al identificarse dicha relación, la información es de utilidad para el tratamiento y la recuperación del paciente. Material y métodos: Estudio retrospectivo y longitudinal durante un período de dos meses; se incluyó a 242 pacientes con lesiones tomográficas consistentes con COVID-19, quienes se analizaron estadísticamente con la prueba ji cuadrada $\left(\chi^{2}\right)$ para determinar la relación con el aumento del diámetro del TAP y mediante partición de tablas para buscar significancia en tomografías indicativas de COVID-19 y divididos en los grados leve, moderado y grave. Resultados: Se calculó la $\chi^{2}$ de los pacientes consistentes con SARS-CoV-2 y TAP > $30 \mathrm{~mm}$ y se obtuvo un valor experimental superior al crítico; por lo tanto, se aceptó que existe significancia estadística; se realizó un segundo análisis mediante partición de tablas y se identificó significancia estadística grave de la infección por SARS-CoV-2 con TAP > $30 \mathrm{~mm}$. Conclusiones: Existe relación estadísticamente significativa entre los pacientes con una TAC en grado de afectación grave por COVID-19 con un diámetro del TAP > 30 mm.

Palabras clave: COVID-19. Arteria pulmonar. Virus del síndrome respiratorio agudo grave. SARS-COV-2. Hipertensión arterial pulmonar 


\section{ABSTRACT}

Objective: Identify in a sample of patients with a diagnosis of infection by SARS-Cov-2, the relationship of COVID-19 with the tomographic data of Pulmonary Arterial Hypertension (PAH), when presenting a Pulmonary Artery Trunk (PAT) caliber greater than $30 \mathrm{~mm}$ as an indirect manifestation of PAH; If this relationship is established, our information will be useful for the treatment and recovery of the patient. Material and methods: Retrospective and longitudinal study, in a period of two months; We included 242 patients with tomographic lesions compatible with COVID-19, who were statistically analyzed with the chi-square test $\left(\chi^{2}\right)$, to establish the relationship with the increase in the diameter of the TAP and by partitioning tables to search for significance, in CT compatible with COVID-19 divided into mild, moderate and severe degrees. Results: $\chi^{2}$ is calculated for patients compatible with SARS-CoV-2 and TAP $>30 \mathrm{~mm}$, obtaining an experimental value higher than the critical value, therefore, we accept that there is statistical significance; We performed a second analysis, by partitioning tables, finding statistical significance in the severe degree of the SARS-CoV-2 infection with TAP $>30 \mathrm{~mm}$. Conclusions: There is a statistically significant relationship between patients with a CT with a severe degree of COVID-19 involvement with a TAP diameter $>30 \mathrm{~mm}$.

Key words: COVID-19. Pulmonary artery. Severe acute respiratory syndrome virus. SARS-CoV-2, CT. Pulmonary arterial hypertension

\section{INTRODUCCIÓN}

La infección por coronavirus (COVID-19) del 2019 es una enfermedad infecciosa causada por el coronavirus del síndrome respiratorio agudo grave de tipo 2 (SARS-Cov-2). El COVID-19 afecta en primer lugar al sistema respiratorio, por lo que en casos sospechosos es habitual solicitar como primera prueba de imagen una radiografía de tórax. Su rendimiento diagnóstico en los estadios iniciales de la enfermedad es limitado, ya que se ha descrito que es posible no detectar hallazgos patológicos en la radiografía que sí identifica la tomografía computarizada de tórax $(\mathrm{TCT})^{1}$.

Este hecho y las circunstancias iniciales de la epidemia, cuando la acumulación de casos sospechosos sobrepasó la disponibilidad de pruebas de RT-PCR, y en virtud de la elevada sensibilidad y especificidad de la TCT, llevaron a algunos grupos de trabajo a adoptar la TCT como prueba diagnóstica en ausencia de RT-PCR, lo que llevó a practicar un número sin precedentes de estudios para tratar de caracterizar una enfermedad infecciosa nueva. La TCT obtuvo en estos estudios muy buenos resultados y se observó que los hallazgos patológicos pueden aparecer incluso antes que los síntomas y diagnosticarse en pacientes con falsos negativos iniciales en la RT-PCR ${ }^{2}$. Se concluyó que la TCT era una herramienta muy valiosa para diagnosticar la infección por COVID-19, tanto en la valoración inicial de la afectación pulmonar como en su seguimiento $^{3}$. Durante la observación de estos 
estudios llamó la atención que algunos pacientes experimentaban un aumento del tronco de la arteria pulmonar; en consecuencia: ¿se relacionaba el COVID-19 con el aumento del diámetro del tronco de la arteria pulmonar?

Este artículo tiene como objetivo determinar, en un grupo de pacientes atendidos en el Hospital Regional General Ignacio Zaragoza de la Ciudad de México con diagnóstico de sospecha de COVID-19, si durante su enfermedad mostraron datos tomográficos indirectos de hipertensión arterial pulmonar, indicada por el aumento del diámetro del tronco de la arteria pulmonar $>30 \mathrm{~mm}$. Debe recordarse que los valores normales del tronco pulmonar principal son de $2.8 \mathrm{~cm} \pm 3 \mathrm{~mm}$ y la rama pulmonar principal derecha de $1.8 \mathrm{~cm} \pm 3 \mathrm{~mm}$; por lo tanto, los calibres mayores son indicativos de la afección mencionada $^{4}$ (Fig. 1A-B)

\section{MATERIAL Y MÉTODOS}

En el programa Excel se recolectaron datos mensuales a partir del 1 de abril del 2020 y hasta el 30 de junio del 2020, en una tabla en la que se registró a todos los pacientes que requirieron TCT; se recolectaron los siguientes datos: género, edad, consistencia con COVID-19 y diámetro del tronco de la arteria pulmonar principal, que debía medirse en el plano de su bifurcación en ángulo recto a su eje largo y justo lateral a la aorta ascendente. Cuando es $\geq 29 \mathrm{~mm}$ tiene un VPP de 0.97, un $87 \%$ de sensibilidad y $89 \%$ de especificidad para hipertensión pulmonar, por lo que esta cifra se ha usado para comprobar que las medidas absolutas no son del todo fiables,

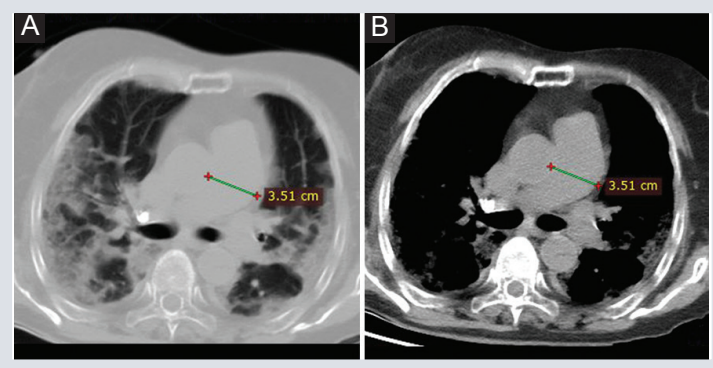

Figura 1. Corte axial de TC con ventana para pulmón (A) y ventana para mediastino (B) a la altura del tronco de la arteria pulmonar que muestra un calibre de $35.1 \mathrm{~mm}$ y afectación del parénquima por infección de COVID-19.

dado que debe tenerse en cuenta que la presión y el tamaño de la arteria pulmonar dependen de la masa corporal, el género y la edad ${ }^{5}$. Existe en la actualidad una propuesta de escala en la evaluación de la gravedad de la enfermedad por TC de tórax, un parámetro semicuantitativo que valora la extensión de lesiones por lóbulos pulmonares, lo que suministra una puntuación de menor a mayor de 1 a 5, con un máximo de 25 puntos respecto del porcentaje de afectación pulmonar; esto proporciona un índice de gravedad que distingue entre leve, moderado y grave; también se revisaron tres categorías respecto del patrón pulmonar predominante (vidrio deslustrado, empedrado, consolidación $)^{6}$.

Se formaron dos grupos denominados "TAC compatible COVID-19 Sí" y "TAC compatible COVID-19 No" (se incluyeron todas las tomografías al margen del grado de gravedad tomográfica para COVID-19 y se excluyeron las tomografías con categoría CO-RADS 3 por presentar duda diagnóstica). A su vez, se subdividieron en tronco de la pulmonar $>30 \mathrm{~mm}$ "Sí" y tronco de la pulmonar 
$>30 \mathrm{~mm}$ "No"; mediante una tabla de $2 \times 2$ con el programa IBM SPSS Statistics se determinó la relación respecto del tronco de la arteria pulmonar durante el periodo determinado. Para el estudio de relación se utilizó la prueba ji cuadrada con nivel de significancia de $0.05 \%$.

Una vez que se demostró la significancia, se realizó un partición de tablas para determinar qué gravedad era la causante de la significancia, por lo que se agrupó a los pacientes consistentes con COVID-19 en leves, moderados y graves, y se subdividieron en tronco de la pulmonar $>30 \mathrm{~mm}$ "Sí" y tronco de la pulmonar $>30 \mathrm{~mm}$ "No". Se realizó una tabla de $3 \times 2$ con el programa IBM SPSS Statistics para establecer la relación respecto del tronco de la arteria pulmonar y se utilizó la prueba ji cuadrada con nivel de significancia del $0.05 \%$. Una vez demostrada la significancia, se excluyó al grado de gravedad y se creó una tabla de 2 x 2 sólo con los pacientes leves y moderados, y se calculó la ji cuadrada para valorar significancia. Con posterioridad se formaron dos grupos, el primero al sumar a los pacientes leves y moderados, y el segundo, de forma independiente, al incluir a los pacientes graves. Se realizó una tabla de $2 \times 2$ con el programa IBM SPSS Statistics, se determinó la relación respecto del tronco de la arteria pulmonar y se usó la prueba ji cuadrada con nivel de significancia del $0.05 \%$.

\section{RESULTADOS}

Se incluyó a 325 pacientes que cumplieron con los criterios establecidos, 193 hombres

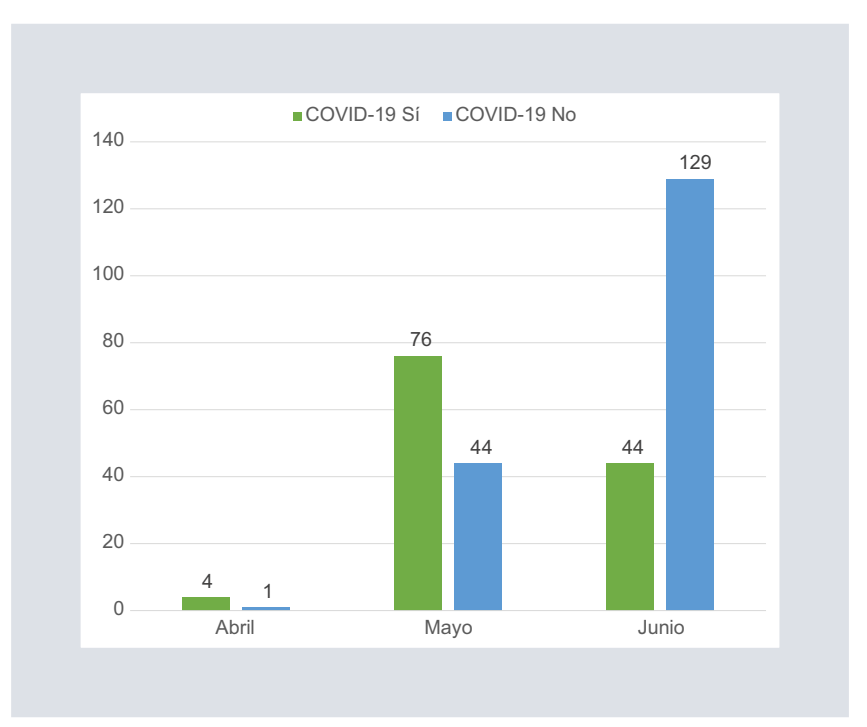

Figura 2. Número de pacientes incluidos en el estudio consistentes y no consistentes con COVID 19, organizados por mes.

(59.4\%) y 132 mujeres (40.6\%) con un intervalo de edad de 15 a 97 años y una media de 51 años (Fig. 2); todos se sometieron a tomografía axial computarizada con protocolo de tórax no óseo en fase simple (Tabla 1).

Se integró un primer grupo de pacientes con tomografía axial computarizada consistente con COVID-19 (CORADS 4,CORADS 5 y CORADS 6) y pacientes con tomografía axial computarizada no consistente con COVID-19 (CORADS 1, CORADS 2 y diagnósticos distintos de neumonía) en relación con el valor del tronco de la pulmonar $<30 \mathrm{~mm}$ y > $30 \mathrm{~mm}$. Se calculó la ji cuadrada para esta tabla de $2 \times 2$ con grados de libertad $v$ $=1$ y $\mathrm{p}=0.05$, con una $\chi_{\text {exp }}^{2}=14.87$, lo que es superior al valor crítico, por lo que se aceptó que hay significancia estadística en el grupo de pacientes con TC consistente con COVID-19 y con tronco de la arteria pulmonar $>30 \mathrm{~mm}$. Por esa razón se llevó a cabo un segundo análisis, esta vez para 
Tabla 1. Número de casos incluidos en el estudio, consistentes y no consistentes con lesiones de COVID-19

\begin{tabular}{|c|c|c|c|c|c|}
\hline & & & \multicolumn{2}{|c|}{$\begin{array}{c}\text { Tronco de la } \\
\text { pulmonar }>30 \mathrm{~mm}\end{array}$} & \multirow[t]{2}{*}{ Total } \\
\hline & & & Sí & No & \\
\hline \multirow[t]{4}{*}{ Consistencia para COVID-19 } & \multirow[t]{2}{*}{ Sí } & Recuento & 79 & 163 & 242 \\
\hline & & $\%$ dentro de tronco de la pulmonar> $30 \mathrm{~mm}$ & $89.9 \%$ & $68.8 \%$ & $74.5 \%$ \\
\hline & \multirow[t]{2}{*}{ NO } & Recuento & 9 & 74 & 83 \\
\hline & & $\%$ dentro de tronco de la pulmonar $>30 \mathrm{~mm}$ & $10.2 \%$ & $31.2 \%$ & $25.5 \%$ \\
\hline \multirow{2}{*}{\multicolumn{2}{|c|}{ Total }} & Recuento & 88 & 237 & 325 \\
\hline & & $\%$ dentro de tronco de la pulmonar $>30 \mathrm{~mm}$ & $100 \%$ & $100 \%$ & \\
\hline
\end{tabular}

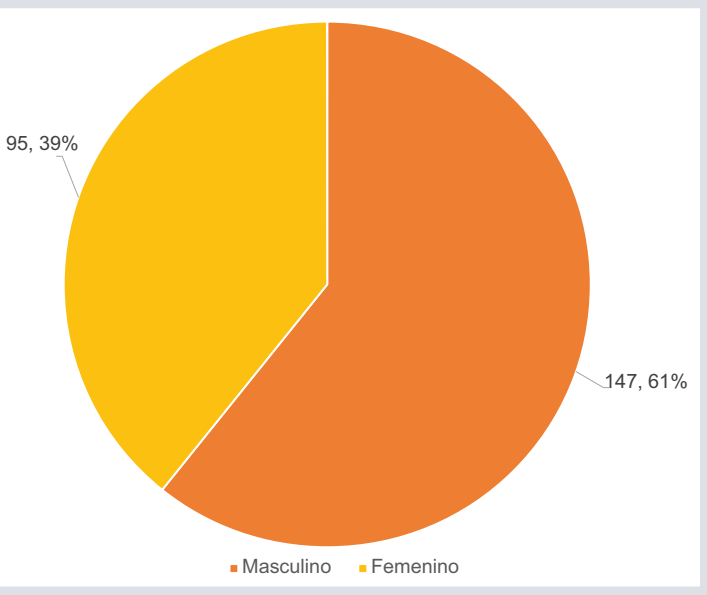

Figura 3. Porcentaje de pacientes por género incluidos en el estudio consistentes con COVID-19 cualquiera que fuera la gravedad.

determinar si la gravedad de la infección guarda relación con el aumento del diámetro del tronco de la arteria pulmonar; en una tabla de $3 \times 2$ se agrupó la gravedad de los pacientes consistentes con COVID-19 (leve, moderado y grave) en relación con el valor del tronco de la pulmonar $>30 \mathrm{~mm}$ y $<30 \mathrm{~mm}$; se obtuvieron 242 casos, de los cuales 147 fueron masculinos (60.7\%) y 95 femeninos (39.3\%) (Fig. 3 y Tabla 2) con grados de libertad $v=2$ y p $=0.05$, y una $\chi_{\text {exp }}^{2}$
= 24.499, lo que supera al valor crítico y por tanto se aceptó que alguno de los grados de gravedad tienen relación con el aumento del tronco de la pulmonar.

Se decidió hacer una partición de tablas, en la cual se excluyó a los pacientes con grado grave de la infección y sólo se analizaron leve y moderado, en relación con el tronco de la arteria pulmonar $>30 \mathrm{~mm} \mathrm{y}<30 \mathrm{~mm}$. Se creó una tabla de $2 \times 2$ (Tabla 3) con grados de libertad $v=1$ y $\mathrm{p}=0.05$, y una $\chi_{\exp }^{2}=1.925$; el valor es menor que el valor crítico y por tanto se asumió que el diámetro del tronco de la arteria pulmonar es independiente de estos dos grados de gravedad.

Por último, se comparó en una tabla de $2 \times 2$ la suma de los pacientes con grado leve + moderado (ya que se demostró que son estadísticamente homogéneos) y los pacientes con grado grave, en relación con el tronco de la arteria pulmonar $>30 \mathrm{~mm} \mathrm{y}<30 \mathrm{~mm}$ (Tabla 4) con grados de libertad $v=1$ y $p=0.05$, y una $\chi_{\text {exp }}^{2}=23.106$, cuyo valor es superior al crítico, por lo que se aceptó que el grado grave es la causa de la significancia estadística en 
TABLA 2. Número de casos incluidos en el estudio, consistentes con lesiones de COVID-19 y categorizados como leves, moderados y graves

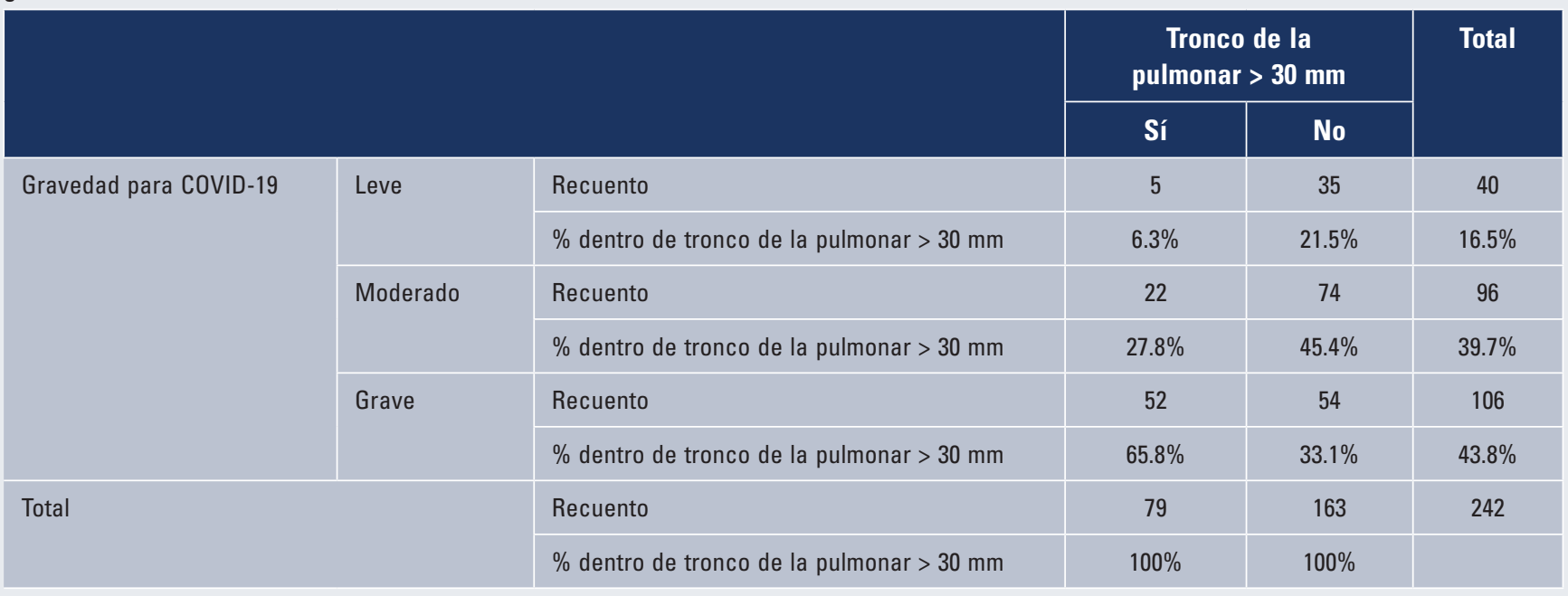

TABLA 3. Número de casos incluidos en el estudio, consistentes con lesiones de COVID-19 y categorizados sólo como leves y moderados

\begin{tabular}{|c|c|c|c|c|c|}
\hline & & & \multicolumn{2}{|c|}{$\begin{array}{c}\text { Tronco de la } \\
\text { pulmonar > } 30 \mathrm{~mm}\end{array}$} & \multirow[t]{2}{*}{ Total } \\
\hline & & & Sí & No & \\
\hline \multirow[t]{4}{*}{ Gravedad para COVID-19 } & \multirow[t]{2}{*}{ Leve } & Recuento & 5 & 35 & 40 \\
\hline & & $\%$ dentro de tronco de la pulmonar $>30 \mathrm{~mm}$ & $18.5 \%$ & $32.1 \%$ & $29.4 \%$ \\
\hline & \multirow[t]{2}{*}{ Moderado } & recuento & 22 & 74 & 96 \\
\hline & & $\%$ dentro de tronco de la pulmonar $>30 \mathrm{~mm}$ & $81.5 \%$ & $67.9 \%$ & $70.6 \%$ \\
\hline \multirow{2}{*}{\multicolumn{2}{|c|}{ Total }} & Recuento & 27 & 109 & 136 \\
\hline & & $\%$ dentro de tronco de la pulmonar $>30 \mathrm{~mm}$ & $100 \%$ & $100 \%$ & \\
\hline
\end{tabular}

relación con el tronco de la arteria pulmonar $>30 \mathrm{~mm}$ (Fig. 4).

\section{DISCUSIÓN}

De acuerdo con los resultados obtenidos se encontró que los grados de gravedad leve y moderado se comportan de forma homogénea $\mathrm{y}$, en consecuencia, el tronco de la arteria pulmonar $>30 \mathrm{~mm}$ no depende de los grados leve y moderado al tener una $\chi^{2}$ menor al valor crítico de acuerdo con la prueba de hipótesis ji cuadrada; en la partición de tablas, el grado grave es la causa de la significancia para tronco de la pulmonar $>30 \mathrm{~mm}$, toda vez que se calculó una $\chi^{2}$ superior al valor crítico de acuerdo con la prueba de hipótesis de la ji cuadrada. 
TabLa 4. Número de casos incluidos en el estudio, consistentes con lesiones de COVID-19 y categorizados como leves y moderados (agrupados) y graves

\begin{tabular}{|c|c|c|c|c|c|}
\hline & & & \multicolumn{2}{|c|}{$\begin{array}{c}\text { Tronco de la } \\
\text { pulmonar }>30 \mathrm{~mm}\end{array}$} & \multirow[t]{2}{*}{ Total } \\
\hline & & & Sí & No & \\
\hline \multirow[t]{4}{*}{ Gravedad para COVID-19 } & \multirow[t]{2}{*}{ Leve + moderado } & Recuento & 27 & 109 & 136 \\
\hline & & $\%$ dentro de tronco de la pulmonar $>30 \mathrm{~mm}$ & $34.2 \%$ & $66.9 \%$ & $56.2 \%$ \\
\hline & \multirow[t]{2}{*}{ Grave } & Recuento & 52 & 54 & 106 \\
\hline & & $\%$ dentro de tronco de la pulmonar $>30 \mathrm{~mm}$ & $65.8 \%$ & $33.1 \%$ & $43.8 \%$ \\
\hline \multirow{2}{*}{\multicolumn{2}{|c|}{ Total }} & Recuento & 79 & 163 & 242 \\
\hline & & $\%$ dentro de tronco de la pulmonar $>30 \mathrm{~mm}$ & $100 \%$ & $100 \%$ & \\
\hline
\end{tabular}

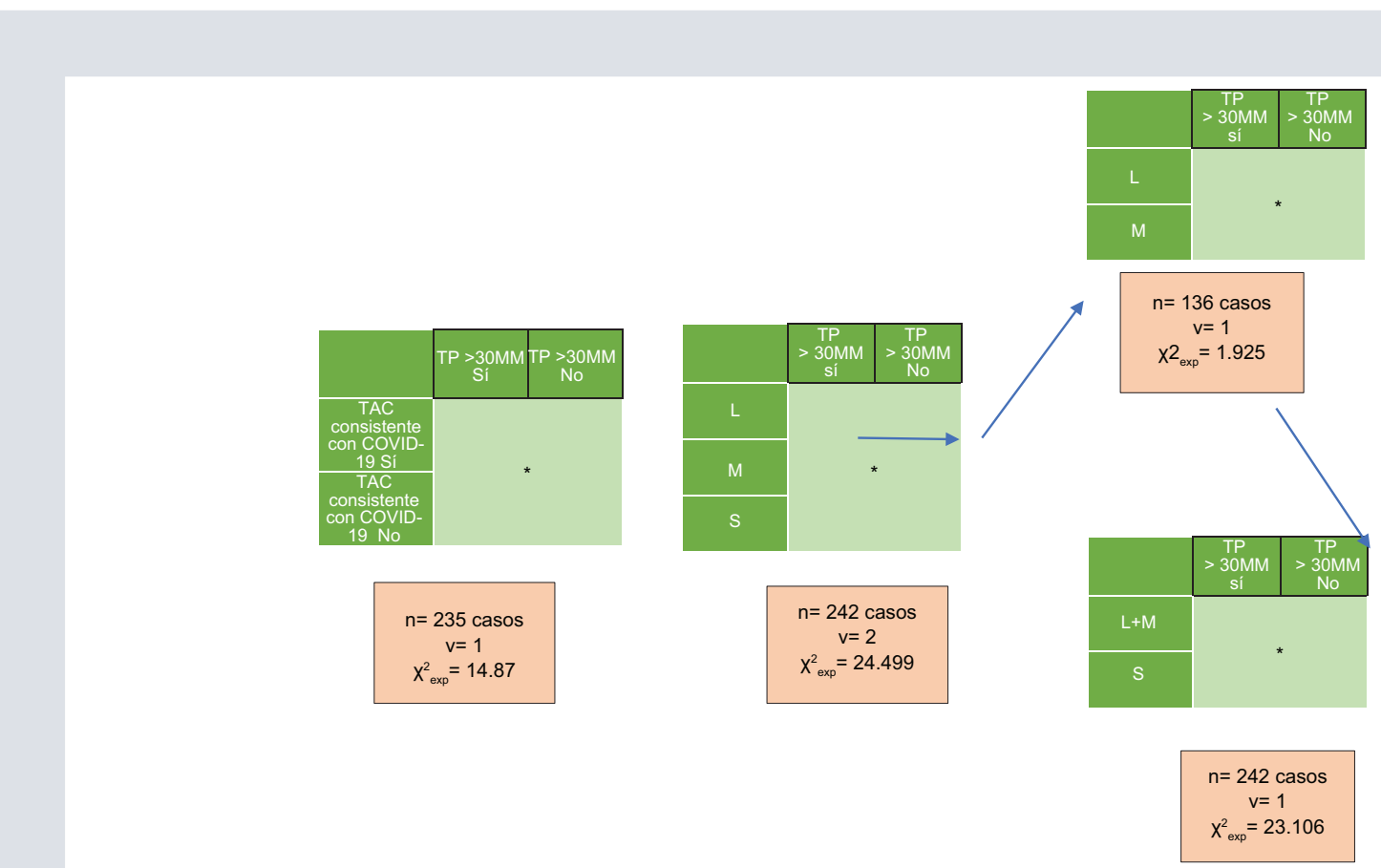

Figura 4. Esquema del análisis estadístico que representa la partición de tablas a través de la ji cuadrada, mediante la cual se encuentra la significancia estadística.

\section{CONCLUSIÓN}

De acuerdo con el análisis estadístico puede concluirse que la proporción de pacientes con tronco de la arteria pulmonar $>30 \mathrm{~mm}$ como dato tomográfico indirecto de hipertensión arterial pulmonar, en pacientes con grado tomográfico grave consistente con COVID-19, es significativamente mayor a la proporción de pacientes con tomografía consistente para 
COVID-19 con grados leve y moderado; es decir, existe una relación estadísticamente significativa entre los pacientes con una tomografía en grado grave por COVID-19 con aumento del diámetro del tronco de la pulmonar $>30 \mathrm{~mm}$.

\section{AGRADECIMIENTOS}

Los autores agradecen al Departamento de Imagenología Diagnóstica y Terapéutica del Hospital Regional General Ignacio Zaragoza, del Instituto de Seguridad y Servicios Sociales de los Trabajadores del Estado por las facilidades concedidas.

\section{CONFLICTO DE INTERESES}

Los autores declaran no tener ningún conflicto de intereses.

\section{RESPONSABILIDADES ÉTICAS}

Protección de personas y animales. Los autores declaran que para esta investigación no se han realizado experimentos en seres humanos ni en animales.

Confidencialidad de los datos. Los autores declaran que han seguido los protocolos de su centro de trabajo sobre la publicación de datos de pacientes.

\section{Derecho a la privacidad y consentimiento} informado. Los autores declaran que en este artículo no aparecen datos de pacientes.

\section{BIBLIOGRAFÍA}

1. Ángel Sánchez-Recalde, Sergio Alcolea, Juan José Ríos-Blanco. Hallazgos por tomografía de coherencia óptica en la hipertensión pulmonar tromboembólica, Revista Española de Cardiología. 10.1016/j.recesp.2014.07.032

2. Imai K, Tabata S, Ikeda M, Noguchi S, Kitagawa Y, Matuoka M, et al. Clinical evaluation of an immunochromatographic IgM/IgG antibody assay and chest computed tomography for the diagnosis of COVID-19 2020 10.1016/j.jcv.2020.104393 Journal of Clinical Virology.

3. Bai H, Hsieh B, Xiong Z. Performance of radiologist in differentiating COVID19 from viral pneumonia on chest CT. Radiology. 2020;Mar 10. Published Online

4. Jiong $\mathrm{Wu}$, Xiaojia $\mathrm{Wu}$, Wenbing Z, Dajing G, Zheng F. Chest $\mathrm{CT}$ findings in patients with coronavirus disease 2019 and its relationship with clinical features. Investigative Radiology. 2020.

5. Avellanas Chavala ML. Hipertensión arterial pulmonar y COVID-19. Med Intensiva. 2020. https://doi.org/10.1016/j.medin.2020.05.005

6. Juárez F, Pensaso Lya. Hallazgos tomográficos en afección pulmonar por COVID-19, Experiencia inicial en el Instituto Nacional de Enfermedades Respiratorias INER, SSA, CDMX, México. 